\title{
STUDENT PREFERENCES WITHIN A HOLISTIC BLENDED LEARNING ENVIRONMENT
}

\author{
Bernice Beukes, Karin Barac, Lynette Nagel, University of Pretoria, South Africa
}

\section{Abstract}

Extant research shows that blended learning environments are widely accepted by students mainly because of the flexibility it offers. However, there is very little research that focuses on students' preferences within a holistic blended learning environment and the contribution that a component makes to the learning of the subject matter, especially in large class settings.

The purpose of this study is to investigate students' perceptions of blended learning components in a holistic blended learning environment and whether these perceptions vary for students with different academic performance levels. A mixed method approach was used in this study performed at a residential university in South Africa and the results indicate that auditing students do have a clear preference for specific components within the environment and significant differences exist between the preferences of different academic performance levels. Such insights allow lecturers to adjust the resources and focus of the different components implemented in a blended learning environment.

\section{Introduction}

Technology enables educators to reach every student in every class every day (Bergmann \& Sams, 2012). How the technology is applied within the educational context impacts on the level of reach into the student's personal learning sphere and in turn, impacts the learning of that student. The literature on blended learning initiatives is widespread. However, research on a holistic blended environment and its components is in short supply. This gap informs the twofold reach questions in this study. How do students perceive the combination of components in a holistic blended learning environment on their learning and whether differences exist between students with varying academic performance levels?

The use of blended learning has proliferated over the years as more and more educators adapt the methodology to enhance student learning. The growing body of research confirms that blended learning environments have been readily accepted by residential students (Bonk \& Graham, 2012; Du, 2011; Garrison \& Kanuka, 2004; Means, Toyama, Murphy, \& Baki, 2013), and that students understand the benefit of having information readily available. However current literature on blended learning tends to focus on either instructional design considerations, comparisons of either purely online, blended or purely face-to-face teaching environments or investigating the effect of one blended learning component implemented in 
the learning environment (Dellaportas \& Hassall, 2013; Dombrowski, Smith, \& Wood, 2013; Massey, Poli, \& Proctor, 2002; Tonge \& Willett, 2012). These studies are also conducted in small to medium settings (less than 150 students) and mostly ignore large class settings (class more than 150 students).

By providing a deeper understanding of blended learning environments, this study aims to expand the literature in two ways. First the study adds a large class setting perspective; second, it presents a multifaceted view on blended learning by examining different components in a holistic blended learning environment. Educators, students and university policymakers can benefit from this research by improving the pedagogy and reaching an effective balance between components for optimum learning

A mixed method approach was used for this study. A survey was administered to third-year undergraduate auditing students at a South African residential university, where a holistic blended learning environment (five components) was implemented in a large class setting. Students had to indicate the perceived level of contribution that each component in the blended learning environment had on their learning of the subject matter on a Likert-type scale and also offer reasons as to how the component contributed to their learning in openended questions. The student data was then analysed overall and also by performance level groups (high, medium and low), based on an average for the formative assessments obtained to determine whether differences in preferences between these groups exist.

The results indicate clear preferences as to which components were perceived to contribute more to student learning. Two distinct groupings within the components in the holistic blended learning environment were identified. In general, students perceive activities driven by the lecturer (online videos, lecture and tutorial) to contribute more to their learning, compared to the student-driven activities (simulation and peer-review/mentoring). There were significant differences in these preferences between the performance levels and the reasons as to how the components contributed to the learning were more distinct between the groupings.

The next section of this paper presents the literature review. This is followed by the description of the research setting and methods used. Thereafter the results are presented and discussed. The paper concludes by identifying areas for future research.

\section{Literature review}

The body of knowledge on blended learning has grown incrementally over the past 15 years covering an array of topics. These include describing the transformative power of blended learning (Garrison \& Kanuka, 2004), identifying best practices to implement blended learning (Alonso, López, Manrique, \& Viñes, 2005; Singh, 2003) and developing a framework for the design of blended learning environments. A thematic review which identified the main themes of blended learning addressed by scholars in higher education, which was originally performed by Halverson, Graham, Spring, Drysdale, and Henrie (2014) and later expanded on by Pima, Odetayo, Iqbal, and Sedoyeka (2018). It highlights four broad themes which 
represent approximately 70\% of literature published between 2000 and 2016 on blended learning (Pima et al., 2018). The most prominent theme identified relates to instructional design considerations (approximately 30\% of literature). This stream of research, which include different perspectives on teaching models and strategies, best practices, implementation and environment matters, guidance, frameworks and the adoption of blended learning (Pima et al., 2018) indicates that blended learning is becoming a common instructional model in higher education, while educators seek to integrate the strengths of both online and face-to-face learning and blend these two modes of teaching so that they become blurred and seamless transitions between them can be achieved (Garrison \& Kanuka, 2004; Pima et al., 2018).

Although research on the instructional design of blended learning appears comprehensive, little is known about students' preferences within a holistic blended learning environment. Most previous studies have either focused on broad design considerations, or have considered the effect of only one or two components in the blended learning environment (Bonk \& Graham, 2012; Ellis, Goodyear, O’hara, \& Prosser, 2007).

It is important to understand what is meant by learning in this context. The definition by Ambrose, Bridges, DiPietro, Lovett, and Norman (2010; p.3) that learning is "a process that leads to change, which occurs as a result of experience and increases the potential for improved and future learning" encapsulates all three core elements (behaviourism, cognitivism and constructivism) of learning theory and is applied as a benchmark for learning in this study. Within this complex construct, blended learning leans more to the constructivism paradigm in that students should construct their own knowledge through experience (Bates, 2016). Therefore, active learning activities are commonly incorporated into a blended learning environment, which includes experiential learning, problem-based learning and cooperative learning activities (Watkins \& Beckem II, 2012; Donnelly, 2010). Deciding which components to incorporate into a blended learning environment vary and is largely influenced by the objective of the blended learning approach and the environment in which the learning takes place. However, it is reasonable to expect that students may have preferences for particular components within a blend and that these preferences will differ within the cohort of students in a class.

Academic performance has been widely used by scholars as a benchmark to determine the effectiveness of educational interventions (Asarta \& Schmidt, 2017; Hun, Loy, \& Hansaram, 2013; McKenzie \& Schweitzer, 2001; Schmulian \& Coetzee, 2011), and as a differentiation mechanism to determine if students with different academic performance capabilities experience a teaching intervention differently (Owston, York, \& Murtha, 2013). The premise for this differentiation is that students with different capability levels approach their studies differently and also learn differently (Honicke \& Broadbent, 2016). Asarta and Schmidt (2017) explained that when students were divided into high, medium or low performing groups, based on their grade point average (GPA), the low performance group achieved higher performance in the traditional approach, compared to the blended learning approach, while the medium group did not indicate any significant difference in their final grades. 
Sanford (2017) found similar results with low achieving students but contradicts the result on medium and high achieving students, stating that all students benefit more from face-to-face formats. Owston et al. (2013) considered satisfaction, convenience, engagement and learning in a blended format based on achievement, and their results are in line with that of Asarta and Schmidt (2017). They found that high achieving students were more satisfied with the blended format. These students also experienced it as more convenient, felt more engaged and believed that they understood key concepts better with the blended format (Asarta \& Schmidt, 2017). The aforementioned studies offer insights into students' preferences, but since these studies are comparisons between face-to-face and online learning, it does not take cognisance of student preferences within a holistic blended learning environment, a notion pointing towards the relevance of our study.

\section{Research setting and methods}

The study was conducted at a South African university with scheduled weekly contact sessions between students and lecturers. The student cohort is culturally diverse, with approximately a third English first language speakers, a third Afrikaans first language speakers and a third indigenous African languages speakers.

The study focuses on an auditing module presented to students in their third year (final year) of specialised accounting undergraduate degree programming. Since 2015, this module was presented in a blended learning environment which was adjusted in 2016 to a holistic blend with a more coherent integration of the different components. The components implemented in the blended learning environment were (a) a flipped classroom (online videos and lectures), (b) a weekly tutorial, (c) an online simulation and (d) finally peer-mentoring and peer feedback.

For the flipped classroom, theoretical videos (referred to as online videos) explaining the basic concepts were recorded and made available on $\mathrm{Bb}$. Students had to watch the videos as preparation for the lecture. During the lecture, the information in the video was placed in context and elaborated upon if necessary, whilst also adding more active learning activities during the session.

The tutorials required students to prepare case study questions at home and then bring the attempted solution to the tutorial for discussion. For tutorials, students were divided into six smaller groups and the focus of the discussion was on how students could improve the quality of their work by demonstrating appropriate examination techniques.

The simulation (called AuditSIM) provided students with an opportunity to practice theoretical auditing concepts in a practical situation, thus mirroring a real audit client. Students had to access client information through $\mathrm{Bb}$ and perform specific tasks in a group in a wiki throughout the year.

For the peer feedback (called TUTBuddy), students had to complete a case study question and then exchange their attempted solution with another group member (the same groups used in 
the simulation were used for the peer feedback). The group member then had to review the attempted solution and provide comments with regard to examination technique displayed and presentation. After completion, students had to complete an online logbook reflecting on the experience. With the peer-mentoring (called BuddyM), students in their third year of undergraduate study had to mentor a second-year student by sharing experiences and giving advice. A minimum of 14 hours had to be spent with the mentee and again students had to record their engagement by completing an online logbook.

\section{Method and data collection}

This study followed a mixed method research approach. A custom-developed survey instrument was used to collect both quantitative and qualitative data. The instrument had to be custom-developed, as a suitable instrument to address the research question of the study could not be identified. In order to expedite completion of the survey, the first part (part A) of the survey (quantitative data) was administered online, using Qualtrics (online survey software). The second part (part B) of the survey was open-ended questions and was distributed manually during the last contact session for the year. The quantitative data were subsequently prepared for statistical analysis and analysed using the Statistical Package for Social Sciences (SPSS), while the qualitative data were captured and analysed using AtlasTi.

Part A of the survey instrument consisted of eight demographical and two questions with sub-questions on the blended learning components. A 5-point Likert scale ranging from 1 not at all contributing to 5 - contributing a great deal was used to determine how students perceived each of the components contributed to their learning of the subject matter. In part $\mathrm{B}$ of the survey, students were requested to comment on how each component in the blended learning environment contributed to their mastering of the subject matter.

The population of this study was the students registered for the B Com Accounting Sciences degree and who enrolled for the third-year module at the university where the study was performed in 2016. All these students were exposed to the holistic blended learning environment in the module and in 2016 there were 651 students enrolled for the module.

The final sample size for the research was 461 responses. Table 1 presents a profile of the sample. An overall response rate of $71 \%$ was obtained. The response rate is high for an online questionnaire, as online surveys typically have lower response rates (Cook, Heath, \& Thompson, 2000). The gender distribution mirrors the gender distribution of the population. For both male and female respondents, a 2.5\% - 3\% difference was found. Male respondents in the sample were slightly underrepresented $(38.2 \%$ to the population of $41 \%)$, while female respondents were slightly over-represented (61.6\% to the population of 59\%). Taking into account the high response rate of $71 \%$, as well as the gender distribution of the respondents, the sample appears to be representative of the population. 


\begin{tabular}{lcccc}
\hline & \multicolumn{2}{c}{ Population } & \multicolumn{2}{c}{ Total } \\
& Total & $\%$ & Total & $\%$ \\
\hline Response rate & 651 & $100 \%$ & 461 & $71 \%$ \\
Males & 267 & $41.0 \%$ & 176 & $38.2 \%$ \\
Females & 384 & $59.0 \%$ & 284 & $61.6 \%$ \\
Missing data (gender not indicated) & & & 1 & $0.2 \%$ \\
\hline
\end{tabular}

\section{Results}

In answering the twofold research question (How students perceived the contribution of the components in a holistic blended learning environment on their learning and differences between academic performance levels), descriptive and inferential statistics analysis was performed on part A of the survey. External validity was achieved since the study was performed in a real-life setting and a representative sample was obtained.

The mean scores for the six component questions were calculated and ranked and presented in Table 2.

Table 2: Perception of the level of contribution of blended learning components on respondents' learning

\begin{tabular}{llc}
\hline Reference & Question & Mean (out of a possible 5) \\
\hline Tutorials & Attending the weekly tutorials & 4.08 \\
$\begin{array}{l}\text { Online } \\
\text { videos }\end{array}$ & The theory videos explaining the basic concepts & 3.97 \\
Lectures & Attending the weekly formal lectures & 3.48 \\
TUTBuddy & $\begin{array}{l}\text { Engaging with my TUT Buddy group on the Buddy } \\
\text { questions }\end{array}$ & 2.85 \\
AuditSIM & Completing the tasks on the AuditSim & 2.79 \\
BuddyM & Engaging with my BuddyM mentee & 2.33 \\
\hline
\end{tabular}

Table 2 shows that the first three components displayed higher mean scores (above 3.0) compared to the latter three (means below 3.0). The mean scores suggested that the tutorial appears to be the blended learning component that contributed highly to the learning of the subject matter, obtaining the highest value (4.08). It is followed by the online videos (3.97) and formal lectures (3.48) which are regarded as contributing moderately to the learning (between 3.0 and 3.9) of the subject matter. The other three blended learning components have a mean score of below 3, indicating that respondents perceive these components to contribute in a limited way to the learning of the subject matter.

The responses were divided into three performance level groups. Academic performance for this study was based on the year mark obtained in the module which was presented as a year module. This year mark was calculated by taking both the formative assessments (85\% contribution to mark) completed during the year and other activities such as the simulation, tutorial attendance, Buddy and class activities (15\% contribution to mark) into consideration. 
Students indicated their own year mark on the anonymous survey. The questionnaire provided five intervals for the year mark, 1 - below 50\%, $2-50 \%-57 \%, 3-58 \%-63 \%, 4-$ $64 \%-69 \%$ and $5-70 \%$ and above. These intervals divided the population into approximately five equal sized groups. In order to reduce the number of groups, responses were redistributed as low $(<57 \%)$, medium $(58 \%-69 \%)$ and high $(>70 \%)$ performing students for the analysis. This was based on an analysis showing the year marks were inflated with marks attributed to participation in class activities, tutorial attendance and Buddy activities. When solely based on formative assessments, the marks would be $7 \%-10 \%$ lower. Table 3 sets out the details of the adjusted groups and the gender distribution within these performance level groups.

Table 3: Distribution of respondents' year mark for the module in 2016, after redistribution

\begin{tabular}{|c|c|c|c|c|c|c|c|c|c|}
\hline \multirow[t]{2}{*}{$\begin{array}{l}\text { Performance } \\
\text { group }\end{array}$} & \multirow[t]{2}{*}{ Interval } & \multicolumn{2}{|c|}{ Population } & \multicolumn{2}{|c|}{ Respondents } & \multicolumn{2}{|c|}{$\begin{array}{c}\text { Male* } \\
\text { respondents }\end{array}$} & \multicolumn{2}{|c|}{ Female* respondent } \\
\hline & & Total & $\%$ & Total & $\%$ & Total & $\%$ & Total & $\%$ \\
\hline High & $>70 \%$ & 118 & $18 \%$ & 108 & $24 \%$ & 38 & $35 \%$ & 70 & $65 \%$ \\
\hline Medium & $\begin{array}{c}58 \%- \\
69 \%\end{array}$ & 296 & $46 \%$ & 212 & $46 \%$ & 73 & $35 \%$ & 138 & $65 \%$ \\
\hline Low & $<57 \%$ & 237 & $36 \%$ & 139 & $30 \%$ & 64 & $46 \%$ & 75 & $54 \%$ \\
\hline Missing data & & & & 2 & & 1 & & 2 & \\
\hline Total & & 651 & $100 \%$ & 461 & $100 \%$ & 176 & & 285 & \\
\hline
\end{tabular}

* Percentage of the respondents per category: high, medium and low

The blended learning components were analysed to determine whether students with different academic performance levels perceived the contribution of the components to their learning differently. The results are presented in Table 4 and Figure 1.

Table 4: $\quad$ Mean scores for learning per performance group and blended learning component

\begin{tabular}{lcccccccccccc}
\hline & \multicolumn{2}{c}{ Tutorial } & \multicolumn{2}{c}{ Video } & \multicolumn{2}{c}{ Lecture } & \multicolumn{2}{c}{ TUT Buddy } & \multicolumn{2}{c}{ AuditSIM } & \multicolumn{2}{c}{ BuddyM } \\
& Mean & SD & Mean & SD & Mean & SD & Mean & SD & Mean & SD & Mean & SD \\
\hline High $^{\$}$ & 4.19 & 1.05 & 4.10 & 0.99 & 3.34 & 1.11 & 2.59 & 1.16 & 2.46 & 1.14 & 2.17 & 1.11 \\
Medium $^{\$}$ & 4.16 & 1.06 & 3.99 & 1.07 & 3.51 & 1.21 & 2.88 & 1.08 & 2.66 & 1.15 & 2.33 & 1.12 \\
Low $^{\text {\$ }}$ & 3.89 & 1.15 & 3.83 & 1.14 & 3.52 & 1.20 & 3.01 & 1.09 & 3.24 & 1.15 & 2.46 & 1.13 \\
\hline \# Standard deviation & & & & & & & & & & &
\end{tabular}

In Figure 1, the difference in perceptions between respondents in different performance groups is portrayed graphically making the differences more visible. 


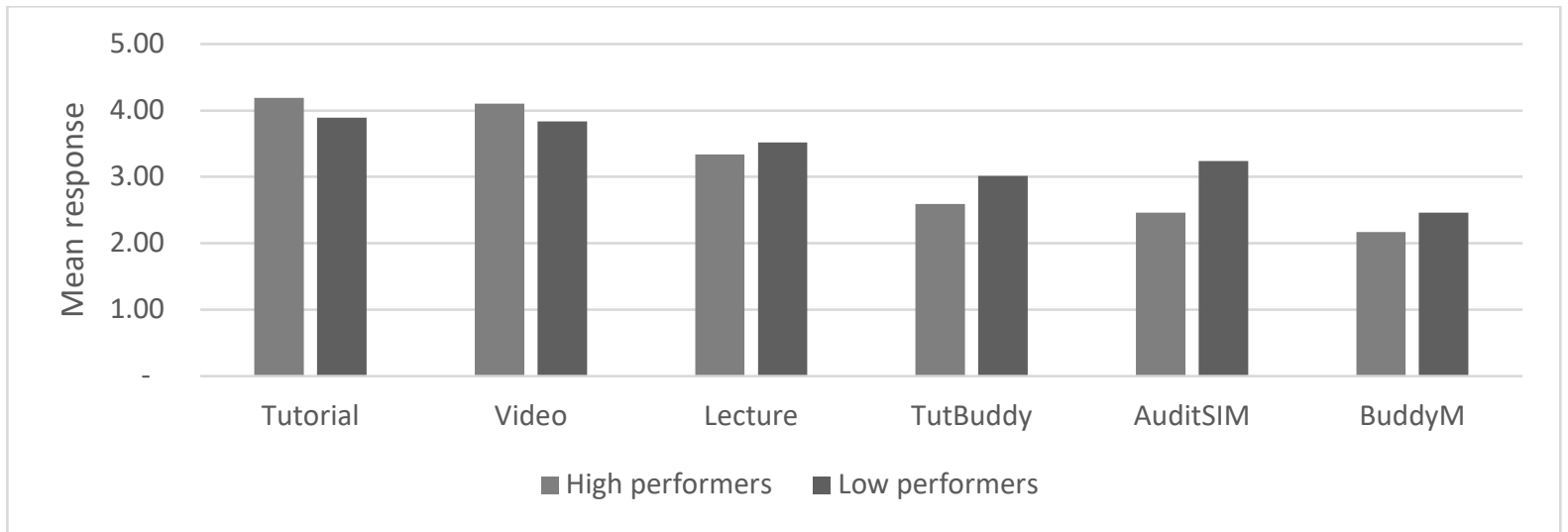

Figure 1. Mean scores for the perception of learning per high and low performance group for each blended learning component

The mean scores of the medium performing respondents followed a consistent pattern between the high and low performing respondents and will therefore not be addressed specifically. The high performing respondents scored their perception of the learning value of tutorials and videos higher compared to the low performing group. This pattern was reversed in the perception of learning in lectures, TUT Buddy, AuditSIM and BuddyM, with the high performers rating those components lower than the low performers. According to the high performers, the tutorial (mean score of 4.19) and videos (mean score of 4.10) contributed highly to their learning, while the formal lectures (mean score of 3.34) contributed moderately. The TUT Buddy, AuditSIM and BuddyM components only had a limited contribution (mean scores of 2.59, 2.46 and 2.17 respectively) to their learning of the subject matter.

Low performing respondents perceived most of the blended learning components to contribute moderately (mean scores between 3.0 and 4.0) to their learning of the subject matter, except for the BuddyM (mean score of 2.46) of which they perceived limited contribution to their learning.

Observing the trends within the groups indicate that the higher the grades, the more the respondents valued the tutorials and videos, and the lower the grades, the more they valued the lectures, TUT Buddy, AuditSIM and BuddyM.

The Kruskal-Wallis test was conducted to determine if statistically significant differences exist between the three performance groups with regard to the blended learning components. The test was used due to the ordinal nature of the data and the results are presented in Table 5.

Table 5: $\quad$ Kruskal-Wallis test results

\begin{tabular}{|c|c|c|c|c|c|c|}
\hline \multicolumn{7}{|c|}{ Test Statistics $\mathrm{a}, \mathrm{b}$} \\
\hline & Videos & Lectures & Tutorials & AuditSIM & BuddyM & TUT Buddy \\
\hline Chi-Square & 3.371 & 1.876 & 6.896 & 28.985 & 4.919 & 7.249 \\
\hline$d f$ & 2 & 2 & 2 & 2 & 2 & 2 \\
\hline Asymp. Sig. & $\begin{array}{l}\text { Not significant } \\
\quad(p=0.185)\end{array}$ & $\begin{array}{l}\text { Not significant } \\
\quad(p=0.391)\end{array}$ & $\begin{array}{c}5 \% \\
(p=0.032)\end{array}$ & $\begin{array}{c}1 \% \\
(p=0.000)\end{array}$ & $\begin{array}{c}10 \% \\
(p=0.085)\end{array}$ & $\begin{array}{c}5 \% \\
(p=0.027)\end{array}$ \\
\hline
\end{tabular}

b Grouping Variable: year mark - high, medium and low performance groups 
The results of Table 5 indicate that statistically significant differences were identified between performance levels. There is a strong statistically significant difference, with regard to the AuditSIM between performance groups $(p=0.000)$ and a statistically significant difference exists at the $5 \%$ level of significance for attending the weekly tutorials $(p=0.032)$ and engaging with the TUT Buddy $(p=0.027)$.

\section{Discussion}

In this section, students' comments are included to deepen the understanding of how the components in the holistic blended learning environment influenced their learning. The study shows students perceived the video, lecture, and tutorial components of blended learning to contribute more to their learning than the other components. They preferred lecturer driven components where the lecturer controls the format and pace of these activities. The AuditSIM and two Buddy components, the more student-driven components that require more active student participation and cooperation with peers, were perceived as less favourable. These components also aimed at developing additional skills (communication, computer and co-operation) that employers require graduates to possess at entry level into the workplace. This finding suggests that students' perceptions of learning are short term orientated (to pass the module), which does not consider their development in generic skills. Based on the mean scores, this tendency was found for high performing respondents who perceived the lecturer driven activities (flipped classroom and tutorial) to contribute more to their learning, whilst the low performing respondents, with the exception of BuddyM, found relatively more value from both the lecturer and student-driven activities than the higher performing students.

The component that all respondents perceived as highly contributing to their learning was the tutorials. Respondents confirmed this preference in the open-ended question, as illustrated by comments "learned a lot" or "I gained the most here" were made by them. Even though research cautions that tutorials should not be the only mode of teaching (Sweeney, O'donoghue, \& Whitehead, 2004), Zhou and Chua (2016) have found in a similar context, that students preferred tutorials above other blended learning interventions. In our study, the number of students per tutorial group (between 60 and 100) was much higher than the norm of one-to-one or one-to-few suggested in the literature (Frey \& Reigeluth, 1986; Sweeney et al., 2004), and therefore not optimal and respondents still perceived tutorials as contributing highly to their learning. They still felt comfortable in a smaller and more informal setting, which allows for frequent questioning (Gordon, 2009). Between the three lecturer-driven components, the tutorial is the element that requires most active participation from students. As this is the preferred element in the holistic blend, this finding is in line with that of Yoder and Hochevar (2005), that active learning activities could improve understanding and performance.

Based on the results, the second and third highest scoring components in the model were also part of the flipped classroom (videos and lecturing) which were perceived as moderately contributing to respondents' learning. The positive view of the flipped classroom agrees with 
Butt (2014), who viewed the concept from an Australian perspective, with actuarial students. Based on mean scores, theoretical videos are in the second position of the different blended learning components. Comments of respondents indicated that they value the fact that videos could be viewed repeatedly, in their own time and that this saved time to gauge an overview of the topics. If therefore appears respondents made use of theoretical videos as and when the need arises, and they developed in more self-regulated learners with the guidance provided in the videos. Respondents perception that the videos contributed more to their learning, is in line with previous research (Gilboy, Heinerichs, \& Pazzaglia, 2015; Little, 2015).

According to Crook and Schofield (2017), it is easy to capture a lecture in digital format and the resultant videos allow for the additional benefits of going back, recapping, note taking and revision. Respondents' perception of a moderate contribution of these components towards their learning could be because videos were seen as an integral part of the lecture. This possibility supports the notion by Gorissen, van Bruggen, and Jochems (2012) that students prefer accompanying online content with their lectures. A factor that could have influenced respondents' perception regarding videos explaining theoretical concepts is their previous exposure to it during their second year in the accounting module.

When comparing the perceptions of the components in the blended learning environment per performance group, more specific differences emerge. With regard to the contribution made for learning, high performing respondents scored the tutorial sessions and videos higher than the low performing respondents, but for the other components, the low performing respondents scored these components higher than the high performers (refer to Table 4 and Figure 1). This difference for the tutorials proved to be statistically significant. A possible explanation is a difference in approach to their studies between the groups. The tutorial requires prior preparation and participation during the session to be effective, and the literature confirms that high performing students are more self-directed learners and willing to work on their own (Owston et al., 2013). This finding is also in line with the tutorial attendance for the population (the class at large), based on weekly attendance registers. The low performing group averaged at $63 \%$ attendance, the medium performing group at $78 \%$ and the high performing group at $87 \%$ attendance. This confirms the high performing respondents' perception that the tutorial sessions contribute to a larger extent to their learning of the subject matter, as compared to their low performing counterparts. This finding, where higher performing students perceived tutorials to highly contribute to their learning, is in line with conclusions reached by Gordon (2009).

In relation to learning, the high performing respondents scored the videos higher, but the lectures lower, compared to the low performing respondents. This indicates that high performing respondents perceived videos explaining the concepts as contributing more to their learning, while this is not the case with formal lectures. The deduction could be made that, as mentioned previously, high performing respondents are self-directed learners who can work on their own (Owston et al., 2013), and have become less dependent on lectures. The fact that the technology element (videos) was scored higher compared to the lectures, confirms the tendency that preference for a specific singular mode of instruction (face-to-face 
only) is declining, and that respondents are becoming more comfortable with alternative modes of instruction, but with available support, a notion supported by the literature (Dziuban \& Moskal, 2011; Kelly, Ponton, \& Rovai, 2007).

The three student-driven components were perceived to contribute moderately to the learning of the low performing respondents but were perceived as limited in contribution for the high performing respondents. Statistically significant differences were identified between the three performance groups with regard to the AuditSIM and the TUT Buddy component. Even though respondents did not perceive these components to contribute highly to their learning of the subject matter, they did realise the benefits that these components could offer in "giving a practical view of how it [an audit] is done" and working in groups and learning from peers. This agrees with the literature on cooperative learning that even though cooperative learning does not impact on students' performance, students are generally positive about the mode of learning (Ballantine \& McCourt Larres, 2009; Johnson, Johnson, \& Smith, 2014; Ravenscroft, Buckless, \& Hassall, 1999). The deduction could thus be made that, regardless of their performance, low performing respondents still perceived the studentdriven components to contribute moderately to their learning. It is also important to recognise that because the low performing respondents also rated the tutorials and videos to contribute more to their learning, they also prefer the blended learning environment, which is in contrast with conclusions reached by Asarta and Schmidt (2017) and Sanford (2017).

\section{Conclusion}

This study sets out to determine how students perceived the contribution of certain components in a blended learning environment on their learning and whether these differed based on academic performance levels. The results indicate that respondents displayed a clear preference for specific components within a blended learning environment and they perceived tutorials to contribute most to their learning of the subject matter.

Significant differences were noted between the different performance groups, and these could be explained because high performing students are more self-directed learners, whilst low performing students scored the student-driven activities higher preferred the social activities more than the high performing counterparts.

The implications of the study are that lecturers can now tailor the environment to suit both high and low performing students and that more autonomy can be given to high performing students, allowing more time to focus on support for low performing students during the contact sessions. Knowing differences exist, lecturers could plan time and resources to allocate to the different blended learning components better, when developing a holistic blended learning environment.

As with any study, there are limitations. This study was performed in a holistic blended learning environment, which might not always be possible to replicate, but the highlights on the individual components and the order of the preferences should make it possible to consider in any blended learning environment. 
This study suggests that a holistic blended learning environment with a balance between lecture driven and student-driven activities covers the needs of high and low performing students. Further research could determine the ideal balance which could guide university policymakers and educators. Future research can repeat the investigation of a holistic blended learning environment but use different variables. For example, the level of technology adoption or comparing preferences between undergraduate and postgraduate modules, in order to determine whether students' maturity could influence the preferences.

\section{References}

Alonso, F., López, G., Manrique, D., \& Viñes, J. M. (2005). An instructional model for webbased e-learning education with a blended learning process approach. British Journal of Educational Technology, 36(2), 217-235.

Ambrose, S. A., Bridges, M. W., DiPietro, M., Lovett, M. C., \& Norman, M. K. (2010). How learning works: Seven research-based principles for smart teaching. John Wiley \& Sons.

Asarta, C. J., \& Schmidt, J. R. (2017). Comparing student performance in blended and traditional courses: Does prior academic achievement matter? The Internet and Higher Education, 32, 29-38. https://doi.org/10.1016/j.iheduc.2016.08.002

Ballantine, J., \& McCourt Larres, P. (2009). Accounting undergraduates' perceptions of cooperative learning as a model for enhancing their interpersonal and communication skills to interface successfully with professional accountancy education and training. Accounting Education: An International Journal, 18(4-5), 387-402.

Bates, T. (2016). Teaching in a digital age. Creative commons - BY-NC. Retrieved from https://www.tonybates.ca/teaching-in-a-digital-age/

Bergmann, J., \& Sams, A. (2012). Flip your classroom: Reach every student in every class every day. International Society for Technology in Education.

Bonk, C. J., \& Graham, C. R. (2012). The handbook of blended learning: Global perspectives, local designs. John Wiley \& Sons.

Butt, A. (2014). Student views on the use of a flipped classroom approach: Evidence from Australia. Business Education \& Accreditation, 6(1), 33-43.

Cook, C., Heath, F., \& Thompson, R. L. (2000). A meta-analysis of response rates in web-or internet-based surveys. Educational and Psychological Measurement, 60(6), 821-836.

Crook, C., \& Schofield, L. (2017). The video lecture. The Internet and Higher Education, 34, 56-64.

Dellaportas, S., \& Hassall, T. (2013). Experiential learning in accounting education: A prison visit. The British Accounting Review, 45(1), 24-36.

https://doi.org/10.1016/j.bar.2012.12.005 
Dombrowski, R. F., Smith, K. J., \& Wood, B. G. (2013). Bridging the education-practice divide: The Salisbury University auditing internship program. Journal of Accounting Education, 31(1), 84-106. https://doi.org/10.1016/j.jaccedu.2012.12.003

Donnelly, R. (2010). Harmonizing technology with interaction in blended problem-based learning. Computers and Education, 54(2), 350-359.

Du, C. (2011). A comparison of traditional and blended learning in introductory principles of accounting course. American Journal of Business Education (AJBE), 4(9), 1-10.

Dziuban, C., \& Moskal, P. (2011). A course is a course is a course: Factor invariance in student evaluation of online, blended and face-to-face learning environments. The Internet and Higher Education, 14(4), 236-241. https://doi.org/10.1016/j.iheduc.2011.05.003

Ellis, R. A., Goodyear, P., O’hara, A., \& Prosser, M. (2007). The University Student Experience of Face-to-Face and Online Discussions: Coherence, Reflection and Meaning. ALT-J: Research in Learning Technology, 15(1), 83-97.

Frey, L. A., \& Reigeluth, C. M. (1986). Instructional Models for Tutoring: A Review. Journal of Instructional Development, 9(1), 2-8.

Garrison, D. R., \& Kanuka, H. (2004). Blended learning: Uncovering its transformative potential in higher education. The Internet and Higher Education, 7(2), 95-105.

Gilboy, M. B., Heinerichs, S., \& Pazzaglia, G. (2015). Enhancing Student Engagement Using the Flipped Classroom. Journal of Nutrition Education and Behavior, 47(1), 109-114. https://doi.org/10.1016/j.jneb.2014.08.008

Gordon, E. E. (2009). 5 Ways to Improve Tutoring Programs. The Phi Delta Kappan, 90(6), 440-445.

Gorissen, P., van Bruggen, J., \& Jochems, W. (2012). Students and recorded lectures: survey on current use and demands for higher education. Research in Learning Technology, 20(3), 17299. https://doi.org/10.3402/rlt.v20i0.17299

Halverson, L. R., Graham, C. R., Spring, K. J., Drysdale, J. S., \& Henrie, C. R. (2014). A thematic analysis of the most highly cited scholarship in the first decade of blended learning research. The Internet and Higher Education, 20(2), 20-34.

Honicke, T., \& Broadbent, J. (2016). The influence of academic self-efficacy on academic performance: A systematic review. Educational Research Review, 17(Supplement C), 6384. https://doi.org/10.1016/j.edurev.2015.11.002

Hun, T. L., Loy, C. K., \& Hansaram, R. M. S. K. (2013). A Study on Predicting Undergraduate's Improvement of Academic Performances based on their Characteristics of Learning and Approaches at a Private Higher Educational Institution. Procedia - Social and Behavioral Sciences, 93(Supplement C), 1957-1965. https://doi.org/10.1016/j.sbspro.2013.10.148 
Johnson, D. W., Johnson, R. T., \& Smith, K. A. (2014). Cooperative learning: Improving university instruction by basing practice on validated theory. Journal on Excellence in University Teaching, 25(4), 1-26.

Kelly, H. F., Ponton, M. K., \& Rovai, A. P. (2007). A comparison of student evaluations of teaching between online and face-to-face courses. The Internet and Higher Education, 10(2), 89-101. https://doi.org/10.1016/j.iheduc.2007.02.001

Little, C. (2015). The flipped classroom in further education: literature review and case study. Research in Post-Compulsory Education, 20(3), 265-279.

Massey, D. W., Poli, P., \& Proctor, R. (2002). The development and evaluation of a team-based audit simulation in the introductory auditing course. Digitalcommons Fairfield University.

McKenzie, K., \& Schweitzer, R. (2001). Who Succeeds at University? Factors predicting academic performance in first year Australian university students. Higher Education Research \& Development, 20(1), 21-33. https://doi.org/10.1080/07924360120043621

Means, B., Toyama, Y., Murphy, R., \& Baki, M. (2013). The effectiveness of online and blended learning: A meta-analysis of the empirical literature. Teachers College Record, 115(3), 1-47.

Owston, R., York, D., \& Murtha, S. (2013). Student perceptions and achievement in a university blended learning strategic initiative. The Internet and Higher Education, 18(3), $38-46$.

Pima, J. M., Odetayo, M., Iqbal, R., \& Sedoyeka, E. (2018). A Thematic Review of Blended Learning in Higher Education. International Journal of Mobile and Blended Learning (IJMBL), 10(1), 1-11.

Ravenscroft, S. P., Buckless, F. A., \& Hassall, T. (1999). Cooperative learning-a literature guide. Accounting Education, 8(2), 163-176.

Sanford, D. (2017). Course format and learning: The moderating role of overall academic performance. The International Journal of Management Education, 15(3), 490-500. https://doi.org/10.1016/j.ijme.2017.08.003

Schmulian, A., \& Coetzee, S. A. (2011). Class absenteeism: Reasons for non-attendance and the effect on academic performance. Accounting Research Journal, 24(2), 178-194.

Singh, H. (2003). Building effective blended learning programs. Educational TechnologySaddle Brook Then Englewood Cliffs NJ-, 43(6), 51-54.

Sweeney, J., O'donoghue, T., \& Whitehead, C. (2004). Traditional face-to-face and web-based tutorials: a study of university students' perspectives on the roles of tutorial participants. Teaching in Higher Education, 9(3), 311-323.

Tonge, R., \& Willett, C. (2012). An audit learning experience: A pilot project through cooperation with a third sector organization. Accounting Education, 21(2), 171-185. 
Watkins, M., \& Beckem II, J. M. (2012). Bringing life to learning: immersive experiential learning simulations for online and blended courses. Journal of Asynchronous Learning Networks, 16(5), 61-71. Retrieved from

https://olj.onlinelearningconsortium.org/index.php/olj/article/view/287

Yoder, J. D., \& Hochevar, C. M. (2005). Encouraging active learning can improve students' performance on examinations. Teaching of Psychology, 32(2), 91-95.

Zhou, M., \& Chua, B. L. (2016). Using Blended Learning Design to Enhance Learning Experience in Teacher Education. International Journal on E-Learning, 15(1), 121-140. 